\title{
ANÁLISE COMPARATIVA DE HÁBITOS DE VIDA DOS UNIVERSITÁRIOS INGRESSANTES E EGRESSANTES DE UMA UNIVERSIDADE PARTICULAR
}

\author{
Elisângela Martins Silva ${ }^{1}$, Marcelo José Alves ${ }^{1}$, Robson Chacon Castoldi ${ }^{1}$, Aline Duarte Ferreira \\ Ceccato $^{1}$, Rômulo Araújo Fernandes ${ }^{2}$, Everton Alex Carvalho Zanuto ${ }^{1}$ \\ ${ }^{1}$ Universidade do Oeste Paulista - UNOESTE, Curso de Educação Física. Presidente Prudente - SP. ${ }^{2}$ Universidade \\ Estadual Paulista - FCT/UNESP, Departamento de Educação Física, Presidente Prudente - SP. E-mail: \\ everton@unoeste.br
}

\section{RESUMO}

Estudo de caráter transversal com características retrospectivas. Objetivo: Verificar o nível de atividade física, estado nutricional, consumo de álcool e tabaco, tempo e qualidade de sono dos universitários ingressantes e egressantes de uma universidade particular de Presidente Prudente/SP. Metodologia: Foram selecionados aleatoriamente 303 alunos de todos os cursos, sendo 172 alunos dos primeiros termos e 131 alunos dos últimos termos respectivamente. Foi realizada entrevista face a face com questionário sobre a prática habitual de atividade física atual e pregressa relatando o tracking da atividade física (infância e adolescência), comportamento sedentário e hábitos alimentares durante comportamento sedentário; Qualidade de sono e fatores de confusão como peso, estatura com IMC calculado a partir destes dados. Resultados: Observou-se que a prevalência de comportamento sedentário foi alta a que corresponde a 50,8\% e que $55,4 \%$ dos universitários possuem sono alterado. Conclusão: Não diferença estatística entre os alunos ingressantes e egressantes.

Palavras-chave: Hábitos Alimentares; Sono; Álcool; Tabaco; Qualidade de Vida.

\section{COMPARATIVE ANALYSIS OF LIFE HABITS OF COLLEGE STUDENTS BEGINNERS AND GRADUATING STUDENTS OF A PRIVATIVE UNIVERSITY.}

\begin{abstract}
Cross-sectional study with retrospective character traits. Objective: To assess the level of physical activity, nutritional status, alcohol consumption and tobacco, time and sleep quality of incoming college students and egressantes a private university in Presidente Prudente/SP. Methodology: Randomly 303 students of all courses, with 172 students of the first terms and the last 131 students were selected terms respectively. Interview was conducted face to face questionnaire on habitual physical activity current and prior reporting the tracking of physical activity (childhood and adolescence), sedentary behavior and dietary habits during sedentary behavior, sleep quality and confounding factors such as weight, height BMI calculated from these data. Results: It was observed that the prevalence of sedentary behavior was high which corresponds to $50.8 \%$ and $55.4 \%$ of the students are sleep changes. Conclusion: There were no statistical differences between freshmen and egressantes.
\end{abstract}

Keywords: Eating Habits; Sleep; Alcohol; Tobacco; Quality of Life. 


\section{INTRODUÇÃO}

A vida de um jovem recémingressante na faculdade muda completamente a ponto do mesmo sofrer uma grande pressão psicológica. Essa pressão psicológica se dá através de decisões e complicações que uma vida acadêmica pode oferecer. Tudo isso pode gerar mudanças no habito alimentar e consequentemente em sua saúde ${ }^{1,2}$.

Por isso, quando adentram na faculdade seus hábitos alimentares e estilo de vida tende a mudar, como: tempo de sono, aumenta o consumo de álcool e cigarro, nível de atividade física, podendo acarretar prejuízos a sua saúde. São reveladas em uma pesquisa as mudanças significativas no perfil dos jovens universitários, enfatizando, principalmente, a falta de prevenção, nutrição inadequada, consumo excessivo de álcool e cigarro e baixo nível de atividade física ${ }^{2}$.

Outro fator que também contribui para essas mudanças é que muitos jovens são influenciáveis, e para serem aceitos em um determinado grupo ou tribo, eles acabam mudando seu estilo simplesmente para passar a pertencer ao grupo ${ }^{3}$.

A família tem um importante papel de influência nos hábitos alimentares dos jovens, tanto que quando eles deixam a casa de seus familiares tendem a deixar seus hábitos saudáveis. A ligação com a família está diretamente ligada à adoção de um estilo de vida saudável, devido a grande cobrança familiar pela adoção de um estilo de vida mais saudável ${ }^{4}$. Jovens que residem fora da casa de seus familiares apresentam composição corporal elevada, nutrição inadequada, como, também, fatores que agravam o risco à saúde, com o excessivo consumo de álcool e baixa prática de atividade física ${ }^{1}$.

A vida universitária faz com que esses jovens adquirem novos relacionamentos, maturidade, maiores raciocínio crítico, uma busca por sua identidade tanto pessoal quanto profissional, tendo que em muitos casos conciliar estudo com trabalho. Tudo isso, pode refletir no estilo de vida e hábitos alimentares de forma a sentirem-se mais libertos a tomarem suas próprias decisões em relação ao que comer, quando, onde e com quem comer ${ }^{5}$.

Segundo a pesquisa feita pelos autores citados acima, os estudantes relataram que ao entrarem na faculdade seus hábitos alimentares foram alterados, estando insatisfeitos com suas dietas alimentares, porém buscam melhorar esta condição com a realização de refeições em horários mais rotineiros, comer em casa, comer alimentos mais saudáveis e diminuir a ingestão dos menos saudáveis que podem trazer prejuízos à própria saúde. 
Pensa-se que pessoas com um nível socioeconômico elevado tivesse mais informações e consciência sobre os benefícios da prática de atividade física e alimentação saudável, mais não foi encontrado numa pesquisa realizada, a qual o nível muito alto de sedentarismo entre os estudantes da área da saúde, a qual era esperada um padrão de atividade física mais elevada por sua classificação socioeconômica corresponder a um nível elevado ${ }^{6}$.

Assim o objetivo deste estudo foi verificar o nível de atividade física, estado nutricional, consumo de álcool e tabaco, tempo e qualidade de sono dos jovens universitários ingressantes e egressantes de uma universidade particular de Presidente Prudente.

\section{METODOS}

\section{Questões éticas}

O estudo foi aprovado pelo Comitê de Ética em Pesquisa Envolvendo Seres Humanos, da Universidade do Oeste Paulista (Número Parecer 390.896). Todos os voluntários assinaram um termo de consentimento livre e esclarecido.

\section{Amostra}

Foram voluntários para esta pesquisa 303 universitários sendo 172 alunos ingressantes e 131 alunos egressantes da Universidade do Oeste Paulista de todos os cursos, arbitrariamente foram selecionados 10 alunos dos primeiros termos e dos últimos termos respectivamente.

\section{Prática de atividades físicas}

Por meio de questionário aplicado na sala de aula, as informações referentes à prática habitual de atividades físicas serão levantadas com a utilização do questionário desenvolvido ${ }^{7}$, validado para a população brasileira $^{8}$.

Foram analisados três construtos dessa prática de atividades físicas durante horários de lazer: intensidade (baixa, moderada e vigorosa), tempo semanal de prática $(<1 \mathrm{~h} / \mathrm{sem} ; 1-2 \mathrm{~h} / \mathrm{sem} ; 2-3 \mathrm{~h} / \mathrm{sem}$; 34h/sem; >4h/sem) e tempo prévio de engajamento (<1 mês; 1-3 meses; 4-6 meses; 7-9 meses; >9 meses). Assim, foram considerados fisicamente ativos os indivíduos que relatarem um mínimo de 180 minutos por semana (3-4h/sem) de atividades físicas de intensidade moderada ou vigorosa, nos últimos quatro meses (4-6 meses). Três categorias foram criadas: (I) indivíduos que não relatarem prática alguma de atividades físicas; (II) indivíduos que relataram menos que 180 minutos por semana / ou intensidade abaixo da estabelecida / ou menor tempo prévio de engajamento; (III) indivíduos que alcançarem o ponto de corte proposto. Este montante semanal de tempo 
é pouco superior aos $150 \mathrm{~min} / \mathrm{sem}$ recomendados atualmente ${ }^{9}$.

\section{Prática de atividades esportivas prévias} (infância e adolescência)

A participação em atividades esportivas durante a infância e a adolescência foi tratada como variável independente. E considerado ativo fisicamente durante a infância (7-10 anos) e a adolescência (11-17 anos) o indivíduo que responderam positivamente a duas perguntas: "Entre os sete e 10 anos, fora da escola, você esteve engajado em alguma atividade esportiva supervisionada, por no mínimo um ano ininterrupto?" e "Entre os 11 e 17 anos, fora da escola, você esteve engajado em alguma atividade esportiva supervisionada, por no mínimo um ano ininterrupto?", respectivamente. Algumas atividades são consideradas como esportivas, por exemplo, futebol, basquetebol, voleibol e handebol e também modalidades de dança (balé, etc.) e ginástica. Com base nessas informações, foi criada uma variável com três categorias: (I) resposta "não" para ambas as perguntas; (II) resposta "sim" para apenas umas das duas perguntas; (III) resposta "sim" para ambas as perguntas.

A presença do tracking da atividade física foi caracterizada por indivíduos fisicamente ativos no lazer ( $\geq 180 \mathrm{~min} / \mathrm{sem}$ ) e que também relatarem a prática de atividades esportivas tanto na infância como na adolescência ${ }^{10}$.

\section{Índice de Massa Corporal}

Algumas variáveis foram elencadas como possíveis fatores de confusão: $O$ peso corporal $(\mathrm{kg})$ e a estatura $(\mathrm{m})$ foram relatados pelos entrevistados e, com base nessas informações, foi calculado o índice de massa corporal (IMC) por meio da divisão do peso corporal pelo quadrado da estatura $\left(\mathrm{kg} / \mathrm{m}^{2}\right)$. A obesidade é definida por valores de IMC $\geq 30 \mathrm{~kg} / \mathrm{m}^{2}$.

\section{Comportamentos sedentários}

Os comportamentos sedentários avaliados foram: número de horas na semana que os jovens permanecem assistindo televisão, usando o computador ou jogando videogame. Foram considerados com comportamento sedentário os jovens que reportaram o somatório do uso de televisão, videogame e computador igual ou superior a 22 horas semanais. Tal ponto de corte foi adotado por estar em consonância com os critérios recomendados por importantes órgãos ${ }^{11}$ e autor ${ }^{12}$.

\section{Hábitos alimentares durante o comportamento sedentário}

Com relação aos hábitos alimentares durante o comportamento sedentário foram abordadas as seguintes questões: 
- Você costuma ingerir alimentos quando está assistindo televisão, usando o computador ou o videogame?

- Em caso de resposta positiva, qual esse tipo de alimento?

- Isso ocorre quantas vezes na semana? Questionário sobre comportamento sedentário e hábitos alimentares durante tal comportamento foram retirados da tese de doutorado ${ }^{12}$.

\section{Qualidade do sono}

A qualidade do sono foi analisada pelo questionário Mini-sleep Questionnaire ${ }^{13}$, validado para o idioma português, o qual é composto por 10 questões com sete possibilidades de respostas (nunca= 1 , muito raramente $=2$, raramente $=3$, às vezes $=4$, frequentemente $=5$, muito frequentemente $=$ 6 e sempre= 7) e fornece um escore adimensional (maior escore, pior a qualidade do sono $)^{14}$. O escore final gerado pelo instrumento pode ser classificado como: sono bom (escore entre 10 e 24 pontos), sono levemente alterado (escore entre 25 e 27 pontos), sono moderadamente alterado (escore entre 28 e 30 pontos) e sono muito alterado (escore acima de 30 pontos). Em nosso estudo, o desfecho utilizado foi o "sono alterado" o qual foi caracterizado por valores $\geq 25$ pontos.

\section{Análise estatística}

Para as variáveis categóricas, a prevalência foi calculada levando em consideração o número de indivíduos com a indicação positiva o desfecho em questão e as demais variáveis analisadas. Os dados foram apresentados em média e desvio padrão, e o teste $T$ independente foram utilizados para comparar o grupo ingressante com o grupo egressante. Valores de significância (p) inferiores a 5\% foram considerados estatisticamente significativos e todas as análises serão realizadas nos software estatístico: SPSS 13.0.

\section{RESULTADOS}

A amostra foi composta por 303 universitários de ambos os sexos (122 masculino [40,3\%] e 181 feminino [59,7\%]), destes $172(56,8 \%)$ ingressantes e $131(43,2 \%)$ egressantes, em sua maioria brancos $(77,2 \%)$, com idade média de 22,8 $\pm 5,1$ anos, altura $1,69 \pm 0,09 \mathrm{~m}$, peso $67,7 \pm 15,3 \mathrm{~kg}$, IMC $23,5 \pm 3,9 \mathrm{~kg} / \mathrm{m}^{2} \quad(71,3 \%$ normal, $21,8 \%$ sobrepeso e 6,9\% obesidade), 157 (51,8\%) trabalhavam, $17(5,6 \%)$ fumantes, 58 (19,1\%) moravam com fumantes, 38 (12,5\%) relataram ingerir bebidas alcoólicas duas ou mais vezes por semana.

A distribuição dos universitários por curso está descrita na tabela I: 
Tabela 1. Distribuição dos voluntários por cursos.

\begin{tabular}{lll}
\hline Variáveis & $\mathrm{N}$ & $\%$ \\
\hline Curso & 17 & \\
Fisioterapia & 18 & 5,6 \\
Engenharia Civil & 14 & 4,6 \\
Medicina & 15 & 4,9 \\
Nutrição & 18 & 5,9 \\
Odontologia & 08 & 2,6 \\
Engenharia de Produção & 10 & 3,3 \\
Biomedicina & 11 & 3,6 \\
Medicina Veterinária & $16:$ & 2,3 \\
Ciência da Computação & 12 & 4,0 \\
Farmácia & 11 & 3,6 \\
Ciências Contábeis & 07 & 2,3 \\
Música & 08 & 2,6 \\
História & 13 & 4,3 \\
Enfermagem & 10 & 3,3 \\
Sistema de Informação & 15 & 5,0 \\
Fonoaudiologia & 03 & 1,0 \\
Matemática & 12 & 4,0 \\
Comunicação Social & 12 & 4,0 \\
Administração & 12 & 4,0 \\
Psicologia & 16 & 5,3 \\
Direito & 13 & 4,3 \\
Química & 13 & 4,3 \\
Educação Física (Bacharel) & 13 & 4,3 \\
Educação & 06 & 2,0 \\
(Licenciatura) & 303 & 100 \\
Ciências Biológicas & & \\
Total & & \\
\hline & & \\
& &
\end{tabular}

A prevalência de pessoas fisicamente ativas foi: $25,1 \%$ na atualidade, $74,3 \%$ na infância, $70 \%$ na adolescência, 18,2\% ao longo da vida (infância+adolescência+atual). A prevalência de comportamento sedentário foi alta $(50,8 \%)$ passam mais de 22 horas por semana em frente à tela (televisão, computador e vídeo game), destes 70,3\% consomem alimentos sendo que $56,1 \%$ preferem consumir alimentos hipercalóricos (chocolates, doces, etc.) durante este comportamento. Sobre a qualidade do sono, $168(55,4 \%)$ foram classificados como sono alterado, 2,3\% necessita de algum acessório para conseguir dormir.

$\mathrm{O}$ teste $\mathrm{T}$ não detectou diferença significativa entre os alunos ingressantes e egressantes, quanto ao IMC (F=2,43, $p=0,120)$, distúrbios do sono $(F=0,123, p=$ 0,717), nível de atividade física no lazer $(F=0,026, \quad p=0,873), \quad$ comportamento sedentário $(F=1,415, p=0,235)$, consumo de tabaco $(F=1,862, p=0,173)$ e álcool $(F=2,624$, $p=0,106)$. Houve diferença estatística apenas para trabalho $(\mathrm{F}=4,06, \quad \mathrm{p}=0,04)$, demonstrando uma superioridade em atividades laborativas dos egressantes.

\section{DISCUSSÃO}

A amostra foi constituída basicamente por mulheres $(59,7 \%)$, brancas $(77,2 \%)$, com média de idade de 22,8 \pm (5,1 anos), apenas 17 (5,6\%) eram fumantes, 58 (19,1\%) moravam com fumantes, 38 (12,5\%) relataram ingerir bebidas alcoólicas duas ou mais vezes por semana. A faixa etária avaliada foi um pouco menor do que encontrado em 871 universitários, que possuíam média de 25 ( \pm 8 anos), sendo $67 \%$ mulheres, o grupo de alunos $>25$ anos apresentou maior prevalência de sedentarismo (38,3\%), tabagismo (10,3\%) e ambos associados $(48,6 \%)$, o total de indivíduos que em algum momento de suas 
vidas foram fumantes (fumantes ocasionais e regulares agrupados a ex-fumantes) foi de 19,5\% ${ }^{15}$. Em outra pesquisa com 194 estudantes do primeiro ano de medicina, $45 \%$ destes relataram beber acima dos limites recomendados para Reino Unido ${ }^{16}$.

Em um estudo transversal analítico cujo tema era "Tabagismo entre acadêmicos da área da saúde", demonstrou que dos 492 alunos entrevistados onde a média de idade foi de $22,20 \pm 4,34$ anos sendo basicamente constituídas por mulheres (60,8\%), brancas $(69,1 \%)$ foi achado uma prevalência de tabagismo de 5,7\%, sendo o estresse a principal razão para o início do habito $(36,8 \%)$, nesta mesma pesquisa ainda foi confirmado que $(58,7 \%)$ consumiam bebida alcóolica $^{17}$. Ainda em relação a consumo de bebida alcóolica, encontrou uma prevalência de $85,3 \%$ dos 285 discentes que se declararam fazer uso de bebidas alcoólicas ${ }^{18}$.

Dos 303 dos estudantes entrevistados $71,3 \%$ teve o IMC de $23,5 \pm 3,9 \mathrm{~kg} / \mathrm{m}^{2}$ considerado normal, $21,8 \%$ sobrepeso e $6,9 \%$ obesidade, já em outra pesquisa ${ }^{19}$ em seu estudo de delineamento transversal de 1039 adultos $\geq 18$ anos encontrou uma prevalência de obesidade $\left(\right.$ IMC $\geq 30 \mathrm{~kg} / \mathrm{m}^{2}$ ) de $17,8 \%$ (IC95\%15,5-20,1), enquanto que a obesidade abdominal (CA $>102 \mathrm{~cm}$ e $>88 \mathrm{~cm}$ para homens e mulheres, respectivamente) esteve presente em 35,1\% (IC95\%32,2-38) dos entrevistados. A obesidade foi mais frequente entre as mulheres - $20,2 \%$ do que entre os homens.

A prevalência de pessoas fisicamente ativas foi de $25,1 \%$ quase a metade do encontrado foi de $51,1 \%$ de pessoas ativas ${ }^{14}$. A qual constatou-se também uma prevalência de $74,3 \%$ na infância, de $70 \%$ na adolescência e 18,2\% ao longo da vida, avaliada 100 alunos maiores de 18 anos estudantes do curso de Educação Física onde 64 eram homens e 36 eram mulheres com idade entre 19 a 45 anos onde foram relatados que $79,5 \%$ destes praticavam atividade física na infância ${ }^{12}$.

O comportamento sedentário encontrado no presente estudo foi considerado alto e insatisfatório (50.8\%), pois está acima das prevalências nacionais e internacionais. Só está abaixo de Portugal que apontou $90 \%$ de pessoas sedentárias ${ }^{20}$, e abaixo do estudo a prevalência de sedentarismo foi estimada em $77,4 \%{ }^{21}$.

Sobre a qualidade do sono, 168 $(55,4 \%)$ foram classificados como sono alterado, 2,3\% necessita de algum acessório para conseguir dormir. Na mesma linha de pesquisa encontrou-se uma qualidade do sono dos 37 estudantes do segundo ano do curso medicina foi considerado adequada (média 3,68\%) ${ }^{22}$. Em equivalência a isso averiguaram o padrão do ciclo sono-vigília de estudantes de medicina que iniciam cedo as aulas, revelou que $38,9 \%$ dos discentes 
tinham qualidade de sono ruim e que a baixa qualidade de sono estava associada com carência e anormalidade do ciclo sonovigília $^{23}$.

Outro fato interessante relatado dos entrevistados, 157 (51,8\%) trabalhava de forma remunerada sendo a maior parte disso alunos egressantes. Ao analisar o estilo de vida de acadêmicos de Educação Física de uma universidade pública do estado de Sergipe com uma amostra de 217 estudantes com média de idade de 20,6 $( \pm 3,1)$ anos, constituindo a maioria do sexo masculino (54,8\%), 67,7\% não trabalhavam de forma remunerada, 57,4\% (IC95\%: 50,7-64,0) não atenderam as recomendações para atividade física, 41,9\% (IC95\%: 35,3-48,5) reportaram uma alimentação inadequada, 1,8\% (IC95\%: 0,1-3,6) fazia uso de tabaco e/ou outros tóxicos, 8,8\% (IC95\%: 4,9-12,5) ingeriam bebidas alcoólicas em excesso, 12,5\% (IC95\%: 8,1-16,9) apresentaram problemas com sono ${ }^{24}$.

As limitações estão relacionadas ao designe do estudo transversal, as principais são o viés da causalidade reversa, onde não podemos afirmar fator causal das prevalências apontadas, e o viés da memória, em perguntas retrospectivas, porém vale ressaltar que a amostra é representativa dentre os universitários da instituição em questão.

\section{CONCLUSÃO}

Por meio desta análise dos hábitos de vida dos universitários, conclui-se que não houve grandes diferenças entre os alunos ingressantes e egressantes. Contudo, faz necessário, maior exploração nesta linha de conhecimento para que possa explicar a real influência da vida acadêmica no estilo de vida dos universitários.

\section{REFERÊNCIAS}

1. Vieira VCR. et al. Perfil socioeconômico, nutricional e de saúde de adolescentes recém-ingressos em uma universidade pública brasileira; Socieconomic, nutritional and health profile of adolescents recently admitted to a Brazilian public university. Rev. nutr, v. 15, n. 3, p. 273-282, 2002.

2. Joia LC. Perfil do estilo de vida individual entre estudantes universitários. Revista Movimenta; v. 3, n. 1, 2010.

3. Engel JF, Blackwell RD, Miniard PW. Comportamento do Consumidor. 8a ed. LTC Editora, 2000, p. 641.

4. Matos M, Carvalhosa S, Fonseca H. O comportamento alimentar dos jovens portugueses. 5, 1. Lisboa: FMH/PEPT/GPT, 2001.

5. Borges CM, Filho DLO. Hábitos alimentares dos estudantes universitários: um estudo qualitativo. Cep, v. 79023, p. 041, 2004.

6. Marcondelli P, Costa THM, Schmitz BAS. Nível de atividade física e hábitos alimentares de universitários do 3으 ao 5o semestres da área da saúde. Rev. Nutr., Campinas, v. 21, n. 1, Feb., 2008.

7. Baecke JA, Burema J, Frijters JE. A short questionnaire for the measurement of 
habitual physical activity in epidemiological studies. Am J ClinNutr. 36: 936-42, 1982.

8. Florindo AA. et al. Methodology to evaluation the habitual physical activity in men aged 50 years or more. Rev. Saúde Pública. $\quad 38(2)$ : 307-14, 2004. http://dx.doi.org/10.1590/S0034-

\section{2}

9. Haskell WL. et al. Physical activity and public health: updated recommendation for adults from the American College of Sports Medicine and the American Heart Association. Med Sci Sports Exerc. 39(8): 1423-34, 2007.

10. Fernandes R.A. Prática da atividade física e prevalência de doenças cardio-metabólicas no estado de São Paulo. Tese (doutorado) apresentada ao Instituto de Biociências, UNESP. Rio Claro, 2011.

11. American Academy of Pediatrics.Committee on Public Education. American Academy of Pediatrics: Children, adolescents, and television. Pediatrics. 107:423-6, 2001. http://dx.doi.org/10.1542/peds.107.2.423

12. Christofaro D.G.D. Excesso de peso em adolescentes: associações com características próprias e de seus pais ou responsáveis. Tese (doutorado) apresentada ao Programa de Pós-Graduação Stricto Sensu em Saúde Coletiva da Universidade Estadual de Londrina, UEL. Londrina, 2012.

13. Zomer J, Pelede AH, Rubin E, Lavie P. Mini-sleep Questionnaire (MSQ) for screening large populations for EDS complaints. In: Koella WP, Rüther E, Schulz H (eds) Sleep '84: Proceedings of the Seventh European Congress on Sleep Research. Fischer, Stuttgart, pp 467-470, 1985.

14. Falavigna A, De Souza-Bezerra ML, Teles AR, Kleber FD, Velho MC, Da Silva RC et al. Consistency and reliability of the Brazilian
Portuguese version of the Mini-Sleep Questionnaire in undergraduate students. Sleep Breath. 15(3): 351-5, 2011. http://dx.doi.org/10.1007/s11325-010-0392$\underline{x}$

15. Cheikll NC, Anamaria FM. "Nível de atividade física e tabagismo em universitários." Rev Saúde Pública 42.4. 6728, 2008.

16. Newbury-Birch D, White $M$, Kamali F. Factors influencing alcohol and illicit drug use amongst medical students. Drug and Alcohol Dependence, 59, 125-130, 2000. http://dx.doi.org/10.1016/S03768716(99)00108-8

17. Garcia G, Ana Flávia, et al. "Tabagismo e fatores associados entre acadêmicos de odontologia." Revista da Faculdade de Odontologia-UPF 14.2, 2010.

18. Amorim, Aline Viana Carvalho, et al. "Álcool e alcoolismo: estudo de prevalência entre discentes do curso de Medicina da UNIFENAS em Belo Horizonte-Minas Gerais." REVISTA MÉDICA DE MINAS GERAIS-RMMG 18.1, 2009.

19. Souza JC. et al. Sonolência diurna excessiva em pré-vestibulandos. J Bras Psiquiatr, v. 56, n. 3, p. 184-7, 2007.

20. Varo JJ, Martínez-González MA, De IralaEstévez J, Kearney J, Gibney M, Martínez JA. Distribution and determinants of sedentary lifestyles in thel European Union. Int J Epidemiol.;32(1):138-46. doi: 10.1093/ije/dyg116, 2003. http://dx.doi.org/10.1093/ije/dyg116

21. Alves JGB. et al. "Prática de esportes durante a adolescência e atividade física de lazer na vida adulta". Rev Bras Med Esporte 11.5. 291-4, 2005.

22. De Almondes KM, John FA. "Padrão do ciclo sono-vigília e sua relação com a 
ansiedade em estudantes universitários." Estudos de Psicologia 8.1. 37-43, 2003.

23. Medeiros, A. L. D., Mendes, D. B. F., Lima, P. F., \& Araujo, J. F. The relationships between sleep-wake cycle and academic performance in medical students. Biological Rhythm Research, 32 (2), 263-270, 2001. http://dx.doi.org/10.1076/brhm.32.2.263.13 $\underline{59}$

24. Silva, Diego Augusto Santos, and Indiara Magalhães Marques Pereira. "Estágios de mudança de comportamento para atividade física e fatores associados em acadêmicos de Educação Física." Revista Brasileira de Atividade Física \& Saúde 15.1. 15-20, 2012.

Recebido para publicação em 13/08/2014

Revisado em 21/08/2014

Aceito em 28/08/2014 